\title{
Fiducial-based image-guided SBRT for pancreatic adenocarcinoma: Does inter-and intra-fraction treatment variation warrant adaptive therapy?
}

Colin S. Hill ${ }^{1 *}$, Sarah Han-Oh ${ }^{1}$, Zhi Cheng ${ }^{1}$, Ken Kang-Hsin Wang ${ }^{1}$, Jeffrey J. Meyer ${ }^{1}$, Joseph M. Herman² and Amol K. Narang ${ }^{1}$

\begin{abstract}
Purpose: Variation in target positioning represents a challenge to set-up reproducibility and reliability of dose delivery with stereotactic body radiation therapy (SBRT) for pancreatic adenocarcinoma (PDAC). While on-board imaging for fiducial matching allows for daily shifts to optimize target positioning, the magnitude of the shift as a result of inter- and intra-fraction variation may directly impact target coverage and dose to organs-at-risk. Herein, we characterize the variation patterns for PDAC patients treated at a high-volume institution with SBRT.

Methods: We reviewed 30 consecutive patients who received SBRT using active breathing coordination (ABC). Patients were aligned to bone and then subsequently shifted to fiducials. Inter-fraction and intra-fraction scans were reviewed to quantify the mean and maximum shift along each axis, and the shift magnitude. A linear regression model was conducted to investigate the relationship between the inter- and intra-fraction shifts.

Results: The mean inter-fraction shift in the LR, AP, and SI axes was $3.1 \pm 1.8 \mathrm{~mm}, 2.9 \pm 1.7 \mathrm{~mm}$, and $3.5 \pm 2.2 \mathrm{~mm}$, respectively, and the mean vector shift was $6.4 \pm 2.3 \mathrm{~mm}$. The mean intra-fraction shift in the LR, AP, and SI directions were $2.0 \pm 0.9 \mathrm{~mm}, 2.0 \pm 1.3 \mathrm{~mm}$, and $2.3 \pm 1.4 \mathrm{~mm}$, respectively, and the mean vector shift was $4.3 \pm 1.8 \mathrm{~mm}$. A linear regression model showed a significant relationship between the inter- and intra-fraction shift in the AP and SI axis and the shift magnitude.

Conclusions: Clinically significant inter- and intra-fraction variation occurs during treatment of PDAC with SBRT even with a comprehensive motion management strategy that utilizes ABC. Future studies to investigate how these variations could lead to variation in the dose to the target and OAR should be investigated. Strategies to mitigate the dosimetric impact, including real time imaging and adaptive therapy, in select cases should be considered.
\end{abstract}

Keywords: SBRT, Pancreatic adenocarcinoma, Active breathing coordination, Motion management, PTV design

\footnotetext{
*Correspondence: chill48@jhmi.edu

1 Department of Radiation Oncology and Molecular Radiation Sciences, Johns Hopkins University School of Medicine, 401 N. Broadway, Suite 1440, Baltimore, MD 21231, USA

Full list of author information is available at the end of the article
}

\section{Introduction}

Pancreatic adenocarcinoma (PDAC) carries a grim prognosis and is estimated to become the second leading cause of cancer death by 2030 [1]. Only half of patients present without clinical evidence of metastatic disease, and only a minority of patients without metastatic disease are amenable to upfront pancreatectomy original author(s) and the source, provide a link to the Creative Commons licence, and indicate if changes were made. The images or other third party material in this article are included in the article's Creative Commons licence, unless indicated otherwise in a credit line to the material. If material is not included in the article's Creative Commons licence and your intended use is not permitted by statutory regulation or exceeds the permitted use, you will need to obtain permission directly from the copyright holder. To view a copy of this licence, visit http://creativecommons.org/licenses/by/4.0/. The Creative Commons Public Domain Dedication waiver (http://creativeco mmons.org/publicdomain/zero/1.0/) applies to the data made available in this article, unless otherwise stated in a credit line to the data. 
at the time of diagnosis due to vascular involvement. Despite the high propensity for distant spread, local progression contributes significantly to the morbidity of the disease. A series of autopsies demonstrated that locally destructive growth may contribute to mortality in a third of patients [2]. Local progression may also significantly contribute to hospitalizations and inhospital mortality, further highlighting the potential importance of local control [3]. With the availability of better systemic therapy regimens, the role of local control has become even more critical [4]. As such, radiation therapy is playing a larger role in the neoadjuvant and definitive setting [5-11]. In the neoadjuvant setting, radiation may improve outcomes with exploration in the setting of vascular involvement, both with respect to margin sterilization and local recurrence risk reduction [11-13]. In the definitive setting, radiation can add to local progression-free survival and prevent local obstructive complications as referenced above.

At our institution, it has been our practice to treat patients with borderline resectable or locally advanced pancreatic cancer with multi-agent chemotherapy for at least 4 months followed by SBRT to 33 Gy (Gy) in 5 fractions, either in the pre-operative or definitive setting $[14,15]$. However, the proximity of radio-sensitive organs, particularly stomach and small and large bowel, makes delivering higher doses per fraction with SBRT challenging. This challenge is compounded further by the fact that the pancreas is a highly mobile organ, susceptible to positional variability with respiratory motion and bowel gas patterns, presenting a significant challenge to accurate targeting with highly conformal treatment [16]. Such variability can be assessed with on-board image guidance, most commonly cone beam computed tomography $(\mathrm{CBCT})$. Due to the limited soft tissue contrast with CBCT, our institution utilizes endoscopically placed fiducials as a surrogate for tumor positioning, which allows for daily shifts off bony anatomy to the fiducials to account for inter-fraction positional variation. Importantly, reports on stereotactic therapy with on-board adaptive planning inform us that daily variation in anatomy can significantly impact the actual dose delivered to the tumor and organs at risk (OAR) [17]. As most sites do not have adaptive therapy capabilities, a better understanding of the magnitude of inter-fraction variation in this clinical setting can help inform appropriate margin design and treatment planning strategies. In addition to appreciating inter-fraction variation, an understanding of intra-fractionation variation in the positioning of these structures is also critical, as both have key implications with respect to margin design, pre-treatment image guidance, and intra-fraction monitoring strategies. Herein, we aim to characterize inter-fraction and intra-fraction variation in tumor positioning in patients treated with stereotactic body radiation therapy (SBRT) for PDAC at a high-volume institution.

\section{Methods \\ Patient selection and treatment course}

We reviewed 30 consecutive patients with borderline resectable or locally advanced PDAC who underwent 5 -fraction SBRT to 33 Gy with volumetric-modulated arc therapy (VMAT) using alpha-cradle (Smithers Medical Products Inc., North Canton, OH, USA) or Vak-lok (CIVCO Medical Solutions, Coralville, IA, USA) immobilization with a wingboard (CIVCO Medical Solutions, Coralville, IA, USA); active breathing coordination (ABC, Elekta, Stockholm, Sweden); hexapod for rotational shifts; and daily image-guidance (IG) with CBCT. Prior to simulation, all patients underwent endoscopic fiducial placement with a goal of implanting three fiducials into the pancreas. For simulation and each fraction of the treatment course, patients were treated with an empty stomach after being nil per os (NPO) for at least 2-3 h. At simulation, patients were instructed to take a deep inspiratory breath hold (DIBH) preferably for $25 \mathrm{~s}$ with $\mathrm{ABC}$ technique, and an intravenous contrast-enhanced planning CT was acquired with the patient in DIBH, with a plan for treatment administration in DIBH.

Target volumes incorporated gross disease with coverage of involved vascular structures. Since 2017, the high-risk nodal basins around the celiac and superior mesenteric arteries have been included in the volume depending on the overall size of the target volume. Fiducials were contoured in the bone window for the purpose of fiducial matching at the time of treatment. VMAT plans were created for each patient. At each treatment fraction, CBCTs were performed to verify patient positioning prior to the start of treatment, whereby patients were aligned first to their bony anatomy and then shifted to the fiducials. Radiation was delivered during the duration of the breath-hold. During treatment, intra-fraction $\mathrm{CBCT}(\mathrm{s})$ were also acquired to confirm target positioning remained consistent throughout treatment, and additional shifts were made to re-align fiducials if needed.

\section{Quantification of the shift and statistical analysis}

The last $\mathrm{CBCT}$ taken prior to treatment initiation for each fraction was selected to characterize the pre-treatment shift off of spine that was required to align fiducials, a measure of inter-fraction variation in tumor positioning. Similarly, the last intra-fraction $\mathrm{CBCT}$ acquired during each fraction was used to characterize intra-fraction variability in tumor positioning. As such, a total of ten CBCTs were reviewed for each patient's treatment course, five pre-treatment and five intra-fraction CBCTs, 
resulting in a total of $300 \mathrm{CBCTs}$ across 30 patients. Utilizing Velocity software (Varian Medical Systems, Inc., Palo Alto, CA, USA) the selected CBCTs for each fraction were fused to the simulation $\mathrm{CT}$ using the bony anatomy of the spine from the simulation as the reference point. The CBCT was then shifted to align the fiducials from the inter-fraction $\mathrm{CBCT}$ with the position of the fiducials on the planning simulation scan, and the shift in the superior-inferior (SI), left-right (LR), and anteriorposterior (AP) axes was then quantified. To quantify the intra-fraction shift, the shift from bone to the fiducials using the intra-fraction СВСТ was subtracted from the inter-fraction shift from bone to the fiducials. The magnitude of the shift was also calculated as a three-dimensional (3D) vector. The mean, maximum, and standard deviation (SD) of the shift in each axis, including the 3D vector, were calculated for 30 individual patients over 5 fractions. In order to provide recommendations for margin design, with the assumption that pre-treatment imaging would account for inter-fraction variation and planning tumor volume (PTV) margin would primarily account for intra-fraction margin, systematic and random components of intra-fraction variation were calculated and combined to form a recommended PTV margin, as has been previously described [18]. Additionally, we also wanted to explore if there was an association between inter-fraction and intra-fraction variation, with the application being that if large inter-fraction variation is appreciated early in the treatment course, this may serve as a predictor of intra-fraction variation as well, which could help inform intra-fraction monitoring strategies and reconsideration of margin design. To explore this relationship, a linear regression analysis with the Pearson correlation test was conducted to investigate the relationship between the pre-treatment shift and the intra-fraction shift.

\section{Results \\ Characterizing inter-fraction variation}

Across 150 pre-treatment CBCTs (five fractions for 30 patients), the mean pre-treatment shift from spine to fiducial in the LR, AP, and SI axes was $3.1 \pm 1.8 \mathrm{~mm}$, $2.9 \pm 1.7 \mathrm{~mm}$, and $3.5 \pm 2.2 \mathrm{~mm}$, respectively, and the average magnitude of the vector shift was $6.4 \pm 2.3 \mathrm{~mm}$. For each patient, maximum shifts were also characterized across the five fractions of treatment. On average, the maximum pre-treatment shift in the LR, AP, and SI axes was $5.5 \pm 2.9 \mathrm{~mm}, 5.1 \pm 2.8 \mathrm{~mm}$, and $6.6 \pm 3.9 \mathrm{~mm}$, respectively, and the average maximum of the magnitude of the vector shift was $9.3 \pm 4.2 \mathrm{~mm}$. Figure 1 presents a summary of the mean pre-treatment shift variation across all three axes and the along the vector.

\section{Characterizing the intra-fraction variation}

Mean intra-fraction shifts were characterized across 150 intra-fraction CBCTs. On average, the mean intrafraction shift in the LR, AP, and SI directions were $2.0 \pm 1.0 \mathrm{~mm}, 2.0 \pm 1.3 \mathrm{~mm}$, and $2.3 \pm 1.2 \mathrm{~mm}$, respectively, and the mean intra-fraction vector shift was $4.3 \pm 1.8 \mathrm{~mm}$. On average, the maximum of the intrafraction shift in the LR, AP, and SI directions were $4.3 \pm 2.1 \mathrm{~mm}, 4.1 \pm 2.8 \mathrm{~mm}$, and $5.3 \pm 3.5 \mathrm{~mm}$, and the maximum of the intra-fraction vector shift was $7.3 \pm 3.8 \mathrm{~mm}$. Figure 2 presents a pictorial summary of the intra-fraction shift variation across all three axes and the along the vector. Utilizing von Herk calculations,

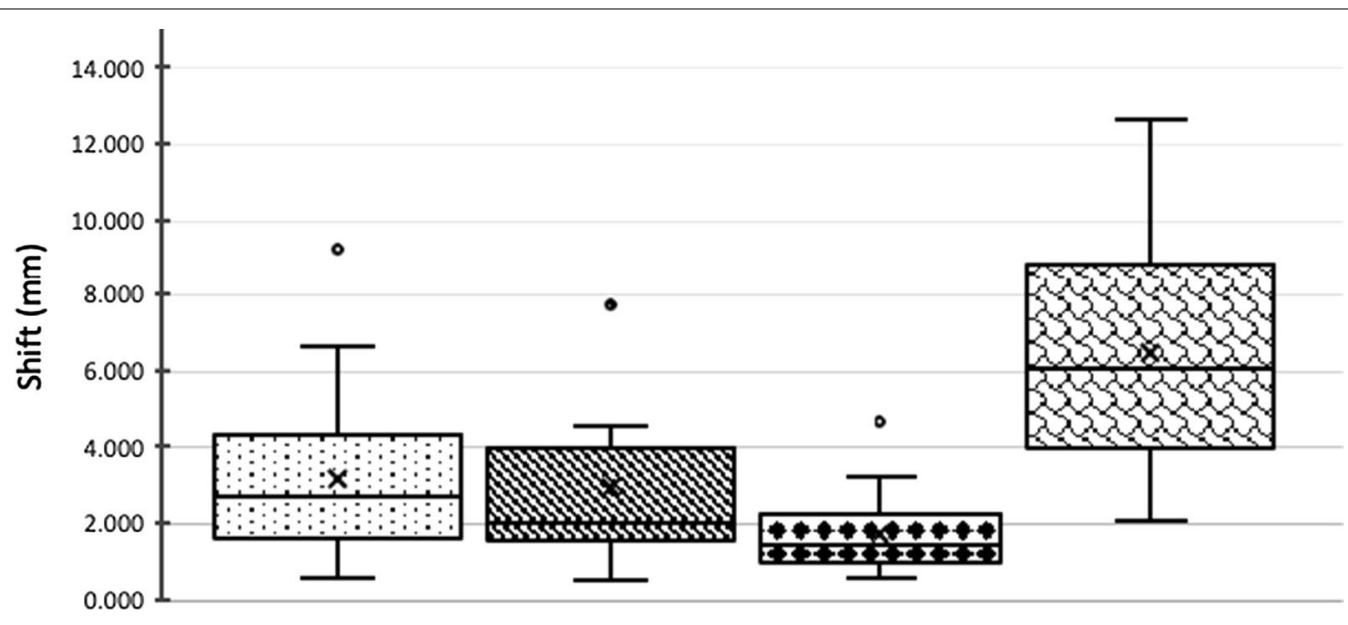

$\boxminus L R \otimes A P \square S I \square$ Magnitude

Fig. 1 Inter-fraction shift variation 


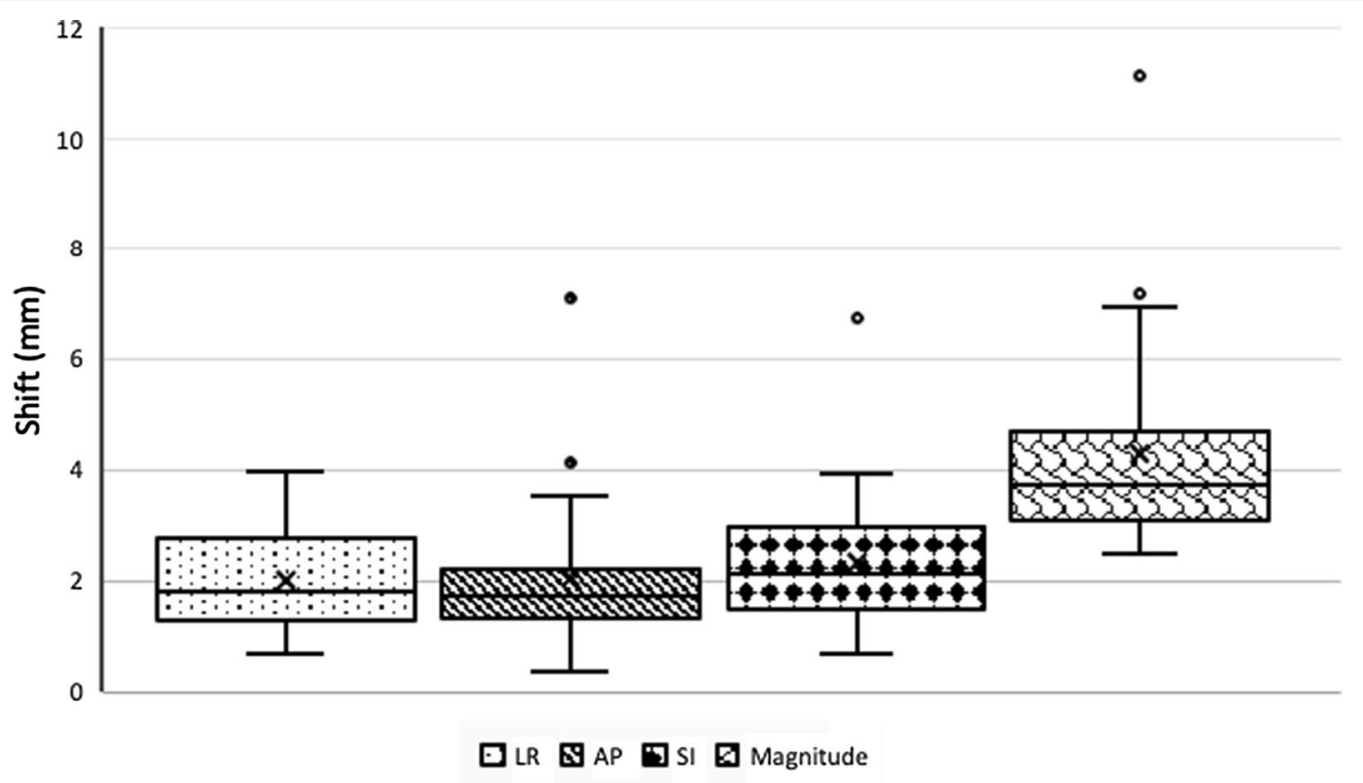

Fig. 2 Intra-fraction shift variation

combining systematic and random components of intrafraction margin results in PTV margin recommendations of $3.7 \mathrm{~mm}$ in the LR axis, $4.4 \mathrm{~mm}$ in the AP axis, and $4.8 \mathrm{~mm}$ in the SI axis [19].

\section{Linear regression relationship of inter- and intra-fraction variation}

A linear regression model was conducted to investigate the relationship in the absolute mean amount of the inter-fraction shift to the absolute mean intra-fraction shift (Table 1, Additional file 1: Fig. S1A-S1D). Along the LR axis, there was no significant relationship between the inter-fraction and the intra-fraction shifts. The shift along the SI and AP axes and the magnitude of the vector shift showed a significant relationship between the inter-fraction and intra-fraction shifts in the model. The shift along the AP axis and for the vector showed moderate correlation between the inter- and intra-fraction shifts $(r=0.57$ and $r=0.55$, respectively). The shifts in the SI axis only showed weak correlation $(r=0.40)$. For example, using the model, a $5 \mathrm{~mm}$ increment in the mean inter-fraction shift along the SI axis would be associated with a $1.8 \mathrm{~mm}$ intra-fraction shift.

\section{Discussion}

Even with a comprehensive motion management strategy with respiratory gating and a breath-hold technique, both inter-fraction and intra-fraction variation remain significant for patients undergoing SBRT for pancreatic cancer. Moreover, for both inter-fraction and intra-fraction variation, the maximum shift, on average, was approximately twice the mean shift, highlighting the wide range of potential variability in tumor positioning that occurs over the course of a treatment course. The dosimetric impact of such variation in the applied shifts are not well understood and may have significant implications for treatment failure and toxicity. Strategies therefore to account for such variation are critical when treating this patient population, as is a better understanding of sources of variation.

To our knowledge, this is the largest series to date in the literature that has characterized inter-fraction and intra-fraction variation in tumor positioning for patients

Table 1 Linear regression relationship between inter- and intra-fraction variation

LR axis Mean abs. Intra-fraction and mean abs. Inter-fraction shift ( $p=0.1228, r=0.288$ )

SI axis Mean abs. Intra-fraction $=0.13 \times($ mean abs. Inter-fraction shift $)+1.18(p=0.0262, r=0.41)$

AP axis Mean abs. Intra-fraction $=0.28 \times($ mean abs. Inter-fraction shift $)+1.17(p=0.001, r=0.57)$

Vector Mean abs. Intra-fraction $=0.25 \times$ (mean abs. Inter-fraction shift $)+2.27(p=0.018, r=0.55)$

Abs. $=$ absolute 
undergoing SBRT for pancreatic cancer with the breathhold technique. Yang et al. reported on eleven patients but only seven patients had pancreatic tumors, and a free-breathing technique was used [20]. Another series reported on five patients treated with intensity modulated radiation for pancreatic cancer and respiratory gating with treatment during the expiration phase [21]. The mean shift off bony anatomy to the fiducial was 1.8, 1.6 , and $4.1 \mathrm{~mm}$ in the LR, AP, and SI directions, respectively, with the SI shift being comparable to our finding $(3.5 \mathrm{~mm})$, but two patients were post-resection so this cohort is not fully representative of patients receiving radiation to an intact pancreas [21]. A comparable study to ours reported on 19 pancreatic cancer patients treated with SBRT using the breath-hold technique, with the mean inter-fraction shift off bone to fiducial of 1.5 , 2.0 , and $3.0 \mathrm{~mm}$ in the LR, AP, and SI axes, respectively [22]. Although treatment verification involved a CBCT and 2-dimensional (2-D) kilo-voltage (kV)-projection images prior to beam delivery on day 1 , the subsequent shifts for fractions 2-5 were measured using breath-hold $\mathrm{kV}$-projection images with the day $1 \mathrm{kV}$-projections serving as the reference point rather than the planning $\mathrm{CT}$ scan [22]. Regarding intra-fraction variation, only a few studies to date that have characterized this for pancreatic tumors with DIBH. Acknowledging its limitations, the aforementioned study reported that the intra-fraction variation with DIBH was within the $2 \mathrm{~mm}$ PTV margin volume [22]. Utilizing end-exhalation breath-holds, Nakamura et al. reported intra-fraction variation along the LR, AP, and SI axes, respectively of $0.0 \pm 1.1 \mathrm{~mm}$, $0.1 \pm 1.2 \mathrm{~mm}$, and $0.1 \pm 1.0 \mathrm{~mm}$ [23]. However, end-exhalation is difficult for patients to achieve compared to a DIBH. Mean intra-fractional variation utilizing real-time tumor tracking report comparable numbers to our study: $0.8 \mathrm{~mm}, 0.6 \mathrm{~mm}$, and $1.7 \mathrm{~mm}$ in the LR, AP, and SI directions, respectively [24].

Inter-fraction variation is assumed to be a combination of several factors such as daily variation in the bowel gas patterns, variation in respiratory-induced movement of the pancreas, and clinical set-up variability. Consistent with our findings, the largest degree of movement has been reported to be in the S-I direction [24-30]. Strategies to address these factors should be considered. With respect to respiratory-induced motion, it is unclear whether there are differences in contribution to interfractional variation between various motion management strategies, including $\mathrm{ABC}$, gating, abdominal compression, or a free-breathing approach. While our institution preferentially favors the $\mathrm{ABC}$ technique, this technique is challenging for patients to master, as it requires them to follow a complex series of synchronized instructions, which are often introduced in a compressed timeframe during simulation. The complexity of the $\mathrm{ABC}$ technique can create anxiety, which may contribute to variation in respiratory motion. Earlier introduction of the $A B C$ technique along with thorough patient education and coaching are important, as may be the development of systems that give real-time visual feedback of the respiratory trace to the patient during treatment. With respect to bowel gas patterns, we now employ a strategy of having patients NPO for five hours prior to treatment to ensure gastric emptying. How to address variation in small and large bowel filling is less clear. Whether scheduled use of simethicone or specific dietary recommendations prior to treatment would be helpful is uncertain, but strict dietary control for this patient population is challenging, as they are often struggling with nutritional deficiency in the setting of pancreatic insufficiency and chemotherapyrelated side effects.

While the lack of resolution on CBCT prevented precise characterization of the dosimetric impact of inter-fraction variation, the experience with magnetic resonance-guided radiation therapy (MRgRT) has suggested that the dosimetric impact on both OARs and target coverage is not insignificant [17]. Notably, within a target volume, there may be varying degrees of interfraction variation, as tumor involvement of relatively rigid structures such as the vasculature may not necessarily exhibit the same degree of movement as the rest of the tumor, a concept that may be particularly true in the setting of elective coverage defined by the peripancreatic vasculature. Indeed, the MRgRT experience has demonstrated the high frequency with which adaptive planning is necessary due to inter-fraction variation. However, recent reports using MRgRT have demonstrated that, with the ability to employ adaptive radiotherapy (ART), safe administration of high biologically equivalent doses (BED) may be feasible, which is encouraging given data suggesting value in dose escalation for this disease. Indeed, some investigators have been able to safely administer treatment schedules with a BED of $100 \mathrm{~Gy}$, which has produced encouraging local control outcomes in other disease sites [31, 32]. In addition to the value that ART may have in this setting, utilization of robust optimization algorithms that account for potential inter-fraction variation, which has primarily been used in the particle therapy space, may also be a consideration $[33,34]$. Planning strategies that help screen for patients exhibiting greater degrees of variation will be useful to determine which patients need adaptive therapy or replanning since this is a resource intensive process [35].

Those factors that contribute to inter-fraction variation can similarly contribute to intra-fraction variation. We postulate that variation in respiratory patterns may be the largest contributor to intra-fraction variation and may 
benefit from the strategies referenced above. Whether bowel gas patterns change significantly during the half hour over which most patients completely treatment is unclear and should be characterized. What is clear is the fact that the magnitude of the intra-fraction variation is not insignificant. Therefore, frequent on-board imaging or continuous monitoring, such as real-time tumor tracking, should be considered to minimize the risk of underdosing target volumes and delivering higher than desired doses to OARs. For patients demonstrating large interfraction variation, our correlation studies emphasize the importance of frequent monitoring for these patients as there may be a correlation with greater degrees of intrafraction variation. Intra-fraction magnitude should also inform appropriate design for target volumes. We suggest taking multiple $\mathrm{ABC}$ scans at simulation, at least three, and then applying an asymmetric expansion for the internal tumor volume to account for the intra-fractional variability with the breath-hold at treatment. As previous studies have shown, a comprehensive understanding of tumor motion can inform margin design [36-38]. On a population level, our analysis suggests that to achieve a 95\% likelihood of tumor coverage while accounting for random and systematic error with intra-fraction variability, the radial for the internal volume with SBRT for pancreatic tumors should be approximately $4.0-4.5 \mathrm{~mm}$ and the SI margin should be approximately $4.5-5.0 \mathrm{~mm}$ [36]. Re-planning should be considered if patients exhibit greater variation with fiducial matching during treatment to minimize the dosimetric impact of this variation, especially with respect to potential toxicity to surrounding organs at risk.

The limitations in our series are inherent to retrospective analysis from a single institution. Although our series is large compared to similar reports in the literature, we are still limited by a small patient cohort. In addition, patients were treated after being NPO only for $2-3 \mathrm{~h}$ and this may have introduced more variability as our practice is now to treat after being NPO for about $5 \mathrm{~h}$. Although we did not appreciate any significant fiducial migration during treatment for our patient cohort, we cannot rule out the possibility of sub-clinical migration. Furthermore, intra-fraction CBCT assessment was acquired only once per fraction and may not fully encapsulate the full range of transitory changes that occurring during treatment. Additionally, when significant, correlation values between inter- and intra-fraction shifts were only weak or moderate.

\section{Conclusion}

Clinically significant inter- and intra-fraction variation occur during treatment of pancreatic cancer with SBRT even with a comprehensive motion management strategy that utilizes the breath-hold technique. Further characterization across motion management strategies should be pursued, as should strategies to mitigate that size and dosimetric impact of this variation.

\section{Abbreviations}

SBRT: Stereotactic body radiation therapy; PDAC: Pancreatic adenocarcinoma; OAR: Organs-at-risk; ABC: Active breathing coordination; SI: Superior-inferior; LR: Left-right; AP: Anterior-posterior; Gy: Gray; CBCT: Cone beam computed tomography; VMAT: Volumetric-modulated arc therapy; IG: Image-guidance; NPO: Nil per OS; DIBH: Deep inspiratory breath hold; 3D: Three-dimensional; SD: Standard deviation; PTV: Planning tumor volume; 2-D: 2-Dimensional; kV: Kilo-voltage; MRGRT: Magnetic resonance-guided radiation therapy; BED: Biologically equivalent dose; ART: Adaptive radiotherapy.

\section{Supplementary Information}

The online version contains supplementary material available at https://doi. org/10.1186/s13014-021-01782-w.

Additional file 1. Linear regression relationship between Inter- and Intrafraction variation in the left-right axis (1A), anterior-posterior axis (1B), superior-inferior axis (1C), and the vector (1D).

\section{Authors' contributions}

CSH analyzed and interpreted the data and was primarily responsible for writing the manuscript. ZC analyzed the data. AKN and SH played a major role in interpreting, analyzing, and writing up the manuscript. All authors read and reviewed the manuscript, and gave approval for submission.

\section{Funding}

This research did not receive any specific grant from funding agencies in the public, commercial, or not-for-profit sectors.

\section{Availability of data and materials}

All data generated or analyzed during this study are included in the published article (and its supplementary information files)

\section{Declarations}

Ethics approval and consent to participate Not applicable.

\section{Consent for publication}

Not applicable.

\section{Competing interests}

The authors declare that they have no competing interests.

\section{Author details}

${ }^{1}$ Department of Radiation Oncology and Molecular Radiation Sciences, Johns Hopkins University School of Medicine, 401 N. Broadway, Suite 1440, Baltimore, MD 21231, USA. ${ }^{2}$ Radiation Medicine, Zucker School of Medicine At Hofstra/Northwell, Lake Success, USA.

Received: 9 September 2020 Accepted: 10 March 2021

Published online: 19 March 2021 


\section{References}

1. Rahib L, Smith BD, Aizenberg R, Rosenzweig AB, Fleshman JM, Matrisian LM. Projecting cancer incidence and deaths to 2030: the unexpected burden of thyroid, liver, and pancreas cancers in the United States. Cancer Res. 2014:74:2913-21.

2. lacobuzio-Donahue CA, Fu B, Yachida S, et al. DPC4 gene status of the primary carcinoma correlates with patterns of failure in patients with pancreatic cancer. J Clin Oncol. 2009;27(11):1806-13. https://doi.org/10.1200/ JCO.2008.17.7188.

3. Cardillo N, Seible DM, Fero KE, Bruggeman AR, Sarkar RR, Azuara A, Simpson DR, Murphy JD. Clinical impact of local progression in pancreatic cancer. J Natl Compr Canc Netw. 2018;16(6):711-7. https://doi.org/10.6004/ jnccn.2018.7013.

4. Gastrointestinal Tumor Study Group Anonymous. Treatment of locally unresectable carcinoma of the pancreas: comparison of combinedmodality therapy (chemotherapy plus radiotherapy) to chemotherapy alone. J Natl Cancer Inst. 1988;80(10):751-5.

5. Chauffert B, Mornex F, Bonnetain F, Rougier P, Mariette C, Bouche O, et al. Phase III trial comparing intensive induction chemoradiotherapy (60 Gy, infusional 5-FU and intermittent cisplatin) followed by maintenance gemcitabine with gemcitabine alone for locally advanced unresectable pancreatic cancer. Definitive results of the 2000-01 FFCD/SFRO study. Ann Oncol. 2008;19(9):1592-9.

6. Klaassen DJ, MacIntyre JM, Catton GE, et al. Treatment of locally unresectable cancer of the stomach and pancreas: a randomized comparison of 5-fluorouracil alone with radiation plus concurrent and maintenance 5-fluorouracil-an eastern cooperative oncology group study. J Clin Oncol. 1985;3:373-8.

7. Conroy T, Hammel P, Hebbar M, Ben Abdelghani M, Wei AC, Raoul J, Choné L, Francois E, Artru P, Biagi JJ, Lecomte T, Assenat E, Faroux R, Ychou M, Volet J, Sauvanet A, Breysacher G, Di Fiore F, Cripps C, Kavan P, Texereau P, Bouhier-Leporrier K, Khemissa-Akouz F, Legoux JL, Juzyna B, Gourgou S, O'Callaghan CJ, Jouffroy-Zeller C, Rat P, Malka D, Castan F, Bachet JB; Canadian Cancer Trials Group and the Unicancer-GI-PRODIGE Group. FOLFIRINOX or gemcitabine as adjuvant therapy for pancreatic cancer. N Engl J Med. 2018;379(25):2395-406. https://doi.org/10.1056/NEJMoa1809 775 .

8. Loehrer PJ Sr, Feng Y, Cardenes H, et al. Gemcitabine alone versus gemcitabine plus radiotherapy in patients with locally advanced pancreatic cancer: an Eastern Cooperative Oncology Group trial. J Clin Oncol. 2011;29(31):4105-12. https://doi.org/10.1200/JCO.2011.34.8904.

9. Herman JM, Chang DT, Goodman KA, et al. Phase 2 multi-institutional trial evaluating gemcitabine and stereotactic body radiotherapy for patients with locally advanced unresectable pancreatic adenocarcinoma. Cancer. 2015;121:1128-37.

10. Hammel P, Huguet F, van Laethem JL, Goldstein D, Glimelius B, Artru P, Borbath I, Bouché O, Shannon J, André T, Mineur L, Chibaudel B, Bonnetain $F$, Louvet $C$. Effect of chemoradiotherapy versus chemotherapy on survival in patients with locally advanced pancreatic cancer controlled after 4 months of gemcitabine with or without erlotinib: the LAP07 randomized clinical trial. JAMA. 2016:315(17):1844-53. https://doi. org/10.1001/jama.2016.4324.

11. Van Tienhoven G, Versteijne $E$, Suker $M$, et al. Preoperative chemoradiotherapy versus immediate surgery for resectable and borderline resectable pancreatic cancer (PREOPANC-1): a randomized, controlled, multicenter phase III trial. JCO. 2018;36(18):LBA4002.

12. Ferrone CR, Marchegiani G, Hong TS, et al. Radiological and surgical implications of neoadjuvant treatment with FOLFIRINOX for locally advanced and borderline resectable pancreatic cancer. Ann Surg. 2015;261:12-7.

13. Faris JE, Blaszkowsky LS, McDermott S, et al. FOLFIRINOX in locally advanced pancreatic cancer: the Massachusetts General Hospital Cancer Center experience. Oncologist. 2013;18:543-8.

14. Rosati L, Hacker-Prietz A, Rao A, et al. Multiagent induction chemotherapy with duration longer than 4 months improves survival in patients with borderline resectable and locally advanced pancreatic adenocarcinoma who received stereotactic body radiation therapy. Int J Radiat Oncol Biol Phys. 2016;96:S205-6.

15. Herman JM, Chang DT, Goodman KA, et al. Phase 2 multi-institutional trial evaluating gemcitabine and stereotactic body radiotherapy for patients with locally advanced unresectable pancreatic adenocarcinoma. Cancer. 2015;121(7):1128-37. https://doi.org/10.1002/cncr.29161.
16. Liu F, Erickson B, Peng C, et al. Characterization and management of interfractional anatomic changes for pancreatic cancer radiotherapy. Int J Radiat Oncol Biol Phys. 2012;83:e423-9.

17. El-Bared N, Portelance L, Spieler BO, Kwon D, Padgett KR, Brown KM, Mellon EA. Dosimetric benefits and practical pitfalls of daily online adaptive MRI-guided stereotactic radiation therapy for pancreatic cancer. Pract Radiat Oncol. 2019;9(1):e46-54. https://doi.org/10.1016/j. prro.2018.08.010.

18. Han-Oh S, Hill C, Kang-Hsin K, Wang KD, Wright JL, Alcorn S, Meyer J, Herman J, Narang A. Geometric reproducibility of fiducial markers and efficacy of a patient-specific margin design using deep inspiration breath hold for stereotactic body radiotherapy for pancreatic cancer. Adv Radiat Oncol. 2021. https://doi.org/10.1016/j.adro.2021.100655.

19. van Herk M, Remeijer P, Rasch C, Lebesque JV. The probability of correct target dosage: dose-population histograms for deriving treatment margins in radiotherapy. Int J Radiat Oncol Biol Phys. 2000;47(4):1121-35. https://doi.org/10.1016/s0360-3016(00)00518-6.

20. Yang W, Fraass BA, Reznik R, Nissen N, Lo S, Jamil LH, et al. Adequacy of inhale/exhale breathhold CT based ITV margins and image-guided registration for free-breathing pancreas and liver SBRT. Radiat Oncol. 2014;9:1-9.

21. Jayachandran P, Minn AY, Van Dam J, Norton JA, Koong AC, Chang DT Interfractional uncertainty in the treatment of pancreatic cancer with radiation. Int J Radiat Oncol Biol Phys. 2010;76(2):603-7. https://doi. org/10.1016/j.jijrobp.2009.06.029.

22. Teboh RF, Srinivasan S, Ng SP, Aliru ML, Herman JM. Setup management for stereotactic body radiation therapy of patients with pancreatic cancer treated via the breath-hold technique. Pract Radiat Oncol. 2019. https:// doi.org/10.1016/j.prro.2019.10.012.

23. Nakamura M, Shibuya K, Shiinoki T, et al. Positional reproducibility of pancreatic tumors under end-exhalation breath-hold conditions using a visual feedback technique. Int J Radiat Oncol Biol Phys. 2011;79:1565-71.

24. Akimoto M, Nakamura M, Nakamura A, Mukumoto N, Kishi T, Goto Y, et al. Inter- and intrafractional variation in the 3-dimensional positions of pancreatic tumors due to respiration under real-time monitoring. Int J Radiat Oncol Biol Phys. 2017;98:1204-11.

25. Bussels B, Goethals L, Feron M, Bielen D, Dymarkowski S, Suetens P, Haustermans K. Respiration-induced movement of the upper abdominal organs: a pitfall for the three-dimensional conformal radiation treatment of pancreatic cancer. Radiother Oncol. 2003;68:69-74.

26. Bhasin DK, Rana SS, Jahagirdar S, Nagi B. Does the pancreas move with respiration? J Gastroenterol Hepatol. 2006;21:1424-7.

27. Mori S, Hara R, Yanagi T, Sharp GC, Kumagai M, Asakura H, et al. Fourdimensional measurement of intrafractional respiratory motion of pancreatic tumors using a 256 multi-slice CT scanner. Radiother Oncol J Eur Soc Ther Radiol Oncol. 2009:92(2):231-7.

28. Gierga DP, Chen GT, Kung JH, Betke M, Lombardi J, Willett CG. Quantification of respiration-induced abdominal tumor motion and its impact on IMRT dose distributions. Int J Radiat Oncol Biol Phys. 2004;58(5):1584-95.

29. Suramo M, Päivänsalo V. MyllyläCranio-caudal movements of the liver, pancreas and kidneys in respiration. Acta Radiol. 1984;25(129):131.

30. Kivisaari L, Makela P, Aarimaa M. Pancreatic mobility: an important factor in pancreatic computed tomography. J Comput Assist Tomogr. 1982;6:854-6.

31. Rudra S, Jiang N, Rosenberg $S A$, Olsen JR, Roach MC, Wan L, Portelance $L$, Mellon EA, Bruynzeel A, Lagerwaard F, Bassetti MF, Parikh PJ, Lee PP. Using adaptive magnetic resonance image-guided radiation therapy for treatment of inoperable pancreatic cancer. Cancer Med. 2019;8(5):2123-32. https://doi.org/10.1002/cam4.2100.

32. Boldrini L, Cusumano D, Cellini F, Azario L, Mattiucci GC, Valentini V. Online adaptive magnetic resonance guided radiotherapy for pancreatic cancer: state of the art, pearls and pitfalls. Radiat Oncol Lond Engl. 2019;14:71.

33. Henke $L$, Kashani $R$, Yang $D$, et al. Simulated online adaptive magnetic resonance-guided stereotactic body radiation therapy for the treatment of oligometastatic disease of the abdomen and central thorax: characterization of potential advantages. Int J Radiat Oncol Biol Phys. 2016;96(5):1078-86. https://doi.org/10.1016/j.jjrobp.2016.08.036.

34. Liang X, Zheng D, Mamalui-Hunter M, Flampouri S, Hoppe B, Mendenhall $\mathrm{N}$, Li Z. ITV-based robust optimization for VMAT planning of stereotactic body radiation therapy of lung cancer. PRO. 2019;9(1):38-48. 
35. Zhang $X$, Rong $Y$, Morrill S, et al. Robust optimization in lung treatment plans accounting for geometric uncertainty. J Appl Clin Med Phys. 2018;19(3):19-26. https://doi.org/10.1002/acm2.12291.

36. Dhont J, Vandemeulebroucke J, Burghelea M, Poels K, Depuydt T, Van Den Begin $\mathrm{R}$, et al. The long- and short-term variability of breathing induced tumor motion in lung and liver over the course of a radiotherapy treatment. Radiother Oncol J Eur Soc Ther Radiol Oncol. 2018;126:339-46.

37. Cusumano D, Dhont J, Boldrini L, Chiloiro G, Teodoli S, Massaccesi $M$, et al. Predicting tumour motion during the whole radiotherapy treatment: a systematic approach for thoracic and abdominal lesions based on real time MR. Radiother Oncol J Eur Soc Ther Radiol Oncol. 2018;129(3):456-62
38. Whitfield G, Jain P, Green M, Watkins G, Henry A, Stratford J, Amer A, Marchant T, Moore C, Price P. Quantifying motion for pancreatic radiotherapy margin calculation. Radiother Oncol. 2012;103(3):360-6. https:// doi.org/10.1016/j.radonc.2012.02.012.

\section{Publisher's Note}

Springer Nature remains neutral with regard to jurisdictional claims in published maps and institutional affiliations.
Ready to submit your research? Choose BMC and benefit from:

- fast, convenient online submission

- thorough peer review by experienced researchers in your field

- rapid publication on acceptance

- support for research data, including large and complex data types

- gold Open Access which fosters wider collaboration and increased citations

- maximum visibility for your research: over $100 \mathrm{M}$ website views per year

At BMC, research is always in progress.

Learn more biomedcentral.com/submissions 